\title{
Complications du Diabète en Côte d'Ivoire chez les Patients Diagnostiques Tardivement
}

\author{
Adjoua Yeboua Florence Kouakou \\ Adama Kamagaté \\ Angoué Paul Yapo
}

Laboratoire de Physiologie animale, de Pharmacologie et de Phytothérapie,

UFR SN, Université Nangui Abrogoua, Abidjan, Côte d'Ivoire

doi: 10.19044/esj.2016.v12n27p250 URL:http://dx.doi.org/10.19044/esj.2016.v12n27p250

\begin{abstract}
Diabetes is a metabolic disorder that affects more and more all social classes. According to the literature this disease have many causes, but some type of diabetes such as Type 2 diabetes is the most linked to a metabolic disorder due to abdominal obesity. To better understand diabetes and its complications, we studied diabetes and its complications in patients who were hospitalized urgently. Our study showed that $95.80 \%$ of hospitalized diabetics are type 2 against $3.36 \%$ type 1 . Among diabetics, there are $56.04 \%$ men and $43.86 \%$ women. But the rate of abdominal obesity was $60.50 \%$ for both men and women. This study also indicated that $60.63 \%$ of hospitalized diabetics suffer of macrovascular complications, against $39.27 \%$ of microvascular complications. Also patients with macrovascular complications suffer specifically from coma, diabetic foot, amputations, stroke; and those with microvascular complications suffer specifically from erectile dysfunction, chronic renal failure, polyneuropathy, lung infections, and also comas. Our study has shown that many patients are unaware of their diabetic condition before the acute complications of diabetes, which is alarming. So we get on the alarm that measures are taken to reduce the complications of diabetes in Ivory Coast.
\end{abstract}

Keywords: Obesity, diabetes, complications, macrovascular, Microvascular.

Résumé

Le diabète est un trouble métabolique qui touche de plus en plus toutes les classes sociales. D'après la littérature cette maladie aurait plusieurs causes, mais certain type de diabète comme le diabète de type 2 serait le plus lié à un désordre métabolique dû à une obésité abdominale. Pour mieux comprendre le diabète et ses complications, nous avons étudié le diabète et 
ses complications chez les patients qui ont été hospitalisés en urgence. Notre étude a montré que $95,80 \%$ des diabétiques hospitalisés sont de type 2 contre $3,36 \%$ de type 1 . Parmi les diabétiques, on dénombre 56,04\% d'hommes et $43,86 \%$ de femmes. On compte $60,50 \%$ d'obésité abdominale pour l'ensemble hommes et femmes. Cette étude nous a aussi indiqué que $60,63 \%$ des diabétiques hospitalisés ont des complications macrovasculaires, et $39,27 \%$ des complications microvasculaires. Aussi les patients avec complications macrovasculaires souffrent de manière spécifique de comas, de pied diabétique, d'amputations, d'AVC ; Et ceux avec les complications microvasculaires souffrent plus spécifiquement de dysfonctionnement érectiles, d'insuffisances rénales chroniques, de polyneuropathies, d'infections pulmonaires, et également de comas. Notre étude a permis de montrer que beaucoup de patients ignorent leur état diabétique bien avant les complications aiguës du diabète, ce qui est alarmant. Donc nous tirons sur la sonnette d'alarme pour que des mesures soient prises pour diminuer les complications du diabète en Côte d'Ivoire.

Mots clés: Obésité, diabète, Complications, Macrovasculaires,
Microvasculaires

\section{Introduction}

Le diabète est un trouble métabolique qui survient lorsque l'organisme est incapable de produire suffisamment d'insuline ou d'utiliser l'insuline de manière efficace (Harris and Zimmet, 1997). Il se caractérise soit par une hyperglycémie chronique, soit une glycémie à jeun supérieure à $1,26 \mathrm{~g} / 1(7 \mathrm{mmol} / \mathrm{l})$ au moins à deux reprises. L'insuline est une hormone fabriquée par les cellules bêta des îlots de Langerhans du pancréas, qui permet au glucose contenu dans les aliments de pénétrer dans les cellules de l'organisme, où il est transformé en énergie nécessaire au bon fonctionnement des muscles et des tissus. Chez une personne atteinte de diabète, le glucose n'est pas absorbé correctement et continue de circuler dans le sang (hyperglycémie), endommageant ainsi peu à peu les tissus. Ces dommages peuvent entraîner des complications mettant la vie de la personne en danger (Fédération Internationale du Diabète [FID], 2013). La classification du diabète a été revue par l'Association Américaine du Diabète (ADA) en 1997 (Guillausseau, 1997), et l'OMS en 1999 (Drouin P. et al., 1999). Actuellement, elle est fondée sur la physiopathologie des différentes formes cliniques et génétiques de la maladie, et non plus sur le traitement reçu (l'Association Américaine du Diabète [ADA], 2012). Le diabète est classé en quatre principaux groupes: le diabète de type 1 (D1), le diabète de type 2 (D2), diabète gestationnel, et autres types spécifiques de diabète peu fréquents et comprenant en compte les affections génétiques, les infections, 
les affections du pancréas exocrine, les endocrinopathies et les causes médicamenteuses.

Le diabète de type 2 est une maladie que l'on peut prévenir, si la résistance à l'insuline est diagnostiquée suffisamment tôt. Des mesures simples modifiant le mode de vie peuvent être très efficaces pour prévenir ou retarder le diabète de type 2 . Les gens doivent ainsi parvenir à un poids corporel normal et le maintenir, faire de l'exercice physique régulier (faire au moins 30 minutes d'exercice, au moins trois fois par semaine), avoir un régime alimentaire adéquat (peu de gras, de sel, de boisson sucrée, plus de fruit et légume par jour), arrêter le tabac et l'alcool. Il faut aussi envisager vérifier sa glycémie le plus souvent. Dans le cas du D1, la prévention est un peu plus complexe.

Ainsi, le nombre d'individus atteints du diabète de type 2 augmente. Le diabète de type 2 est une complication fréquente de l'obésité. On estime que $90 \%$ de l'ensemble des diabétiques sont de types 2. Le D2 représente donc la forme la plus rependue du diabète. Sa fréquence croît aussi bien dans les pays développés que dans les pays en voie de développement. Les estimations de la Fédération Internationale du Diabète (FID) de 2013 indiquent que 382 millions de personnes sont atteintes de diabète dans le monde. Ce nombre est supposé dépasser les 592 millions dans moins de 25 ans. En effet, on estime à 175 millions le nombre de cas de diabétiques non diagnostiqués à l'heure actuelle qui s'exposent à des complications du diabète par ignorance. En Côte d'Ivoire, les estimations de la FID indiquent 501,53 milliers de cas de diabète et 11884 décès liés à cette maladie. Au vu du nombre total de personnes atteintes dans les pays à faible et moyen revenu, où l'épidémie s'amplifie à une vitesse alarmante, les derniers chiffres de la FID donnent une indication inquiétante de l'impact futur du diabète en tant que menace majeure pour le développement mondial (FID, 2013). Le diabète constitue un problème majeur de santé publique, et cette maladie représente la première cause d'insuffisance rénale dans tous les pays occidentaux, d'un quart à un tiers des causes d'infarctus du myocarde, la première cause d'artériopathie des membres inférieurs, et une des grandes causes d'AVC et de cécité (Halimi, 2003).

Les complications chroniques ou dégénératives sont l'ensemble des atteintes viscérales dues aux lésions vasculaires liées à la maladie. Ces lésions peuvent toucher les gros vaisseaux à destinée cérébrale, les coronaires et les artères des membres inférieurs (les macro-angiopathies). Ces lésions peuvent aussi se localiser au niveau des petits vaisseaux qui irriguent la rétine, le rein et les nerfs périphériques (les micro-angiopathies). (Item, 2011).

Tout diabétique peut un jour présenter des Complications métabolites aiguës constituant une urgence thérapeutique. Les diabétiques de type 1 sont 
exposés à deux types de complications métaboliques aiguës, l'acidocétose et l'hypoglycémie. Les diabétiques de type 2 sont exposés au coma hyperosmolaire, mais aussi aux hypoglycémies et à l'acidose lactique.

En Côte d'Ivoire, la prévalence des infections associées au diabète est de $57 \%$ (Lokrou et al., 1987). La susceptibilité aux infections est due à une altération des fonctions leucocytaires liée à l'hyperglycémie. Les plus fréquentes sont : les infections urinaires, infections cutanéo-muqueuses, les infections bucco-dentaires, les infections ORL et les infections pulmonaires.

Afin de mieux comprendre comment se présente cette maladie en Côte d'Ivoire, nous étudierons les complications du diabète entraînant des hospitalisations à partir des signes cliniques que présentent les patients. Plus spécifiquement, nous avons évalué la prévalence de l'obésité sur des malades qui ont été hospitalisés au CHU de Yopougon; Etudier les facteurs anthropométriques, biologiques, socio-économiques et les comportements à risques; Étudier les complications que présentent les malades du diabète en urgence médicale au CHU de Yopougon.

\section{Matériel et méthodes}

Le matériel utilisé dans cette partie du travail se compose de 119 dossiers de malades, une fiche d'enquête et un stylo. L'enquête s'est déroulée au CHU de Yopougon. Il a été question du dépouillement des dossiers de malades hospitalisés pour le diabète. Ces dossiers ont été pris au hasard. Les informations recueillies concernaient les caractéristiques sociodémographiques (sexe, âge), les caractéristiques anthropométriques (poids, taille, indice de masse corporel (IMC), tour de taille), la tension artérielle, le statut socioéconomique, le statut marital, le tabagisme, les antécédents d'hypertension artérielle (HTA), le statut pondérale, et les différentes complications dues au diabète.

\section{Analyse statistique}

Les données ont été saisies à l'aide du logiciel Excel et analysés à l'aide du logiciel statistica 7.1. La comparaison des moyennes a été effectuée par un test de Student. Les tests ont été jugés significatifs au niveau de confiance de $95 \%$ soit $\mathrm{p}<0,05$.

\section{Résultats}

Les résultats de l'étude portant sur les diabétiques hospitalisés sont présentés dans les tableaux suivants (tableau 1, tableau 2, tableau 3 et tableau 4). On a AVC: accident vasculaire cérébral, D: diabète, DE: dysfonctionnement érectiles, HTA: hypertension artérielle, IMC: indice de masse corporel, IRC: d'insuffisances rénales chroniques, N: Nombre, PD: 
pied diabétique, PN: polyneuropathies TT: tour de taille, TAD:

Tension artérielle diastolique, et TAS: Tension artérielle systolique.

Tableau 1 : Caractéristiques sociodémographiques des diabétiques en fonction du sexe

\begin{tabular}{|c|c|c|c|c|c|c|c|}
\hline & \multicolumn{2}{|c|}{ Hommes } & \multicolumn{2}{|c|}{ Femmes } & \multicolumn{2}{|c|}{ Total } & \multirow[t]{2}{*}{$\mathbf{P}$} \\
\hline Type de diabète & $\mathbf{N}$ & $\%$ & $\mathbf{N}$ & $\%$ & $\mathbf{N}$ & $\%$ & \\
\hline $\mathrm{D} 2$ & 64 & 56,64 & 50 & 43,86 & 114 & 95,80 & \\
\hline D1 & 1 & 25 & 3 & 75 & 4 & 3,36 & \\
\hline D pancréatique & 0 & 0 & 1 & 100 & 1 & 0,84 & \\
\hline Total & 65 & 56,30 & 54 & 45,38 & 119 & 100 & \\
\hline \multicolumn{8}{|c|}{ Mesures anthropométriques } \\
\hline Age moyen & \multicolumn{2}{|c|}{$54,96 \pm 1,6$} & 53,52 & $\pm 1,82$ & \multicolumn{2}{|c|}{$54,32 \pm 1,17$} & 0,276 \\
\hline Poids moyen & \multicolumn{2}{|c|}{$70,8 \pm 1,91$} & \multicolumn{2}{|c|}{$66,27 \pm 2,56$} & \multicolumn{2}{|c|}{$68,75 \pm 1,57$} & 0,078 \\
\hline Taille moyenne & \multicolumn{2}{|c|}{$1,72 \pm 0,008$} & \multicolumn{2}{|c|}{$1,62 \pm 0,008$} & \multicolumn{2}{|c|}{$1,67 \pm 0,01$} & 0,0001 \\
\hline IMC moyen & \multicolumn{2}{|c|}{$23,93 \pm 0,58$} & \multicolumn{2}{|c|}{$25,62 \pm 0,89$} & \multicolumn{2}{|c|}{$24,68 \pm 0,52$} & 0,055 \\
\hline TT moyen & \multicolumn{2}{|c|}{$91,22 \pm 1,52$} & \multicolumn{2}{|c|}{$90,09 \pm 2,12$} & \multicolumn{2}{|c|}{$90,71 \pm 1,26$} & 0,331 \\
\hline TAS moyen & \multicolumn{2}{|c|}{$13,4 \pm 0,34$} & \multicolumn{2}{|c|}{$13,17 \pm 0,38$} & \multicolumn{2}{|c|}{$13,3 \pm 0,25$} & 0,326 \\
\hline TAD moyen & \multicolumn{2}{|c|}{$7,91 \pm 0,19$} & \multicolumn{2}{|c|}{$7,79 \pm 0,22$} & \multicolumn{2}{|c|}{$7,86 \pm 0,15$} & 0,339 \\
\hline \multicolumn{8}{|c|}{ Statut socioéconomique } \\
\hline$<1500000 /$ an & \multicolumn{2}{|c|}{$11(16,92 \%)$} & \multicolumn{2}{|c|}{$39(72,22 \%)$} & \multicolumn{2}{|c|}{$50(42,02 \%)$} & \\
\hline$\geq 1500000 / \mathrm{an}$ & \multicolumn{2}{|c|}{$54(83,08 \%)$} & \multicolumn{2}{|c|}{$15(27,78 \%)$} & \multicolumn{2}{|c|}{$69(57,98 \%)$} & \\
\hline & & & u d'il & truction & & & \\
\hline$<\mathrm{BEPC}$ & 32 & $9,23 \%)$ & 42( & ,78\%) & 74 & $18 \%)$ & \\
\hline$\geq \mathrm{BEPC}$ & 33 & $0,77 \%)$ & 12( & $2,22 \%)$ & 45( & $82 \%)$ & \\
\hline Tabagisme & & & & & & & \\
\hline Fumeur & & $31 \%)$ & & 0 & & $72 \%)$ & \\
\hline Non-fumeur & 54 & $3,08 \%)$ & & $00 \%)$ & 108 & $0,75 \%)$ & \\
\hline Ancien fumeur & & $62 \%)$ & & 0 & & $2 \%)$ & \\
\hline & & Ant & dents & ersonnt & & & \\
\hline HTA & 20 & $0,77 \%)$ & 22 & $, 74 \%)$ & 42 & $, 29 \%)$ & \\
\hline Macrosomie & & & 16( & $, 63 \%)$ & & & \\
\hline
\end{tabular}

Les différents types de diabète rencontrés sont :

- $\quad 95,80 \%$ de D2 dont 56,64\% d'hommes et 43,36\% de femmes ;

- $\quad 3,36 \%$ de D1;

- $\quad 0,84 \%$ d'un autre type de diabète (diabète pancréatique).

Dans l'ensemble, l'âge de ces diabétiques varie de 18 mois à 81 ans avec une moyenne de 54,62 ans. Toutefois, l'âge des diabétiques de type 2 étudiés varie de 29 à 81 ans avec une moyenne de 55,58 ans alors que celui des D1 varie de 18 mois à 31 ans avec une moyenne de 19,96 ans.

Le poids moyen retrouvé dans cet échantillon est de $68,75 \pm 1,57 \mathrm{~kg}$. Celui des hommes étant de 70,8 $\pm 1,91 \mathrm{~kg}$ et celui des femmes $68,27 \pm 2,56 \mathrm{~kg}$.

La taille moyenne est de $1,67 \pm 001 \mathrm{~m}(1,71 \pm 0,008 \mathrm{~m}$ chez 1 'homme contre $1,62 \pm 0,008 \mathrm{~m}$ chez la femme).

L'IMC moyen est de $24,68 \pm 052 \mathrm{~kg} / \mathrm{m}^{2}$. Il semble plus élevé chez les femmes que chez les hommes $(25,62$ vs 23,92$)$ mais la différence n'est pas significative $(\mathrm{p}=0,055)$. 
Le tour de taille moyen dans notre échantillon est de 90,71 $\pm 1,26 \mathrm{~cm}$ sans différence significative selon le sexe $(\mathrm{p}=0,331)$.

L'analyse du niveau d'instruction met en évidence que $72,22 \%$ n'ont pas le BEPC. Selon le sexe, il s'agit de $77,78 \%$ des femmes enquêtées et de 49,23\% des hommes enquêtés.

Globalement, la plupart des diabétiques ont un niveau socioéconomique supérieur à 1,5 millions FCFA par an (57,98\%). Mais, selon le sexe, il s'agit de la plupart des hommes $(83,08 \%)$. Quant aux femmes, elles ont pour la plupart un niveau socio-économique bas.

En ce qui concerne le tabagisme, $90,76 \%$ de la population enquêtée n'a jamais fumé, $2,52 \%$ a fumé dans le passé et $6,72 \%$ sont fumeurs. Chez 1'homme, $83,08 \%$ déclarent n'avoir jamais fumé et $12,31 \%$ fument. La population féminine enquêtée n'a jamais fumé.

$35,29 \%$ de ces diabétiques pris au hasard ont des antécédents de HTA. La fréquence est plus importante chez les femmes que chez les hommes (40,74\% vs 30,77\%). On note aussi que $29,63 \%$ des femmes ont des antécédents de macrosomie.

Tableau 2 : Etude du statut pondéral des hommes et des femmes diabétiques en fonction de la tranche d'âge

\begin{tabular}{|c|c|c|c|c|c|c|}
\hline AGE (ans) & $\leq 39$ & $40-49$ & $50-59$ & $60-69$ & $\geq 70$ & Total \\
\hline \multicolumn{7}{|c|}{ Hommes } \\
\hline \multicolumn{7}{|c|}{ Par rapport à l'IMC } \\
\hline Obèse & 0 & $2(11,11 \%)$ & $4(20 \%)$ & 0 & 0 & $8(12,31 \%)$ \\
\hline Surpoids & $\begin{array}{c}2 \\
(33,33 \%)\end{array}$ & $7(38,89 \%)$ & $7(55 \%)$ & $4(36,36 \%)$ & $5(45,45 \%)$ & $31(47,69 \%)$ \\
\hline Normal & $\begin{array}{c}1 \\
(16,67 \%)\end{array}$ & $7(41,18 \%)$ & $7(35 \%)$ & $4(36,36 \%)$ & $4(36,36 \%)$ & $23(35,88 \%)$ \\
\hline Maigre & $3(50 \%)$ & $1(5,88 \%)$ & $2(10 \%)$ & $3(27,27 \%)$ & $2(18,18 \%)$ & $11(16,92 \%)$ \\
\hline \multicolumn{7}{|c|}{ Par rapport à TT } \\
\hline Obèse & 0 & $11(64,71 \%)$ & $12(60 \%)$ & $3(27,27 \%)$ & $6(54,55 \%)$ & $32(49,23 \%)$ \\
\hline Non obèse & $6(100 \%)$ & $6(35,29 \%)$ & $8(40 \%)$ & $8(72,72 \%)$ & $5(45,45 \%)$ & $33(50,77 \%)$ \\
\hline \multicolumn{7}{|c|}{ Femmes } \\
\hline \multicolumn{7}{|c|}{ Par rapport à l'IMC } \\
\hline Obèse & 0 & $2(14,29 \%)$ & $6(40 \%)$ & $5(41,67 \%)$ & $2(28,57 \%)$ & $15(27,28 \%)$ \\
\hline Surpoids & $2(33,33 \%)$ & $5(35,71 \%)$ & $3(20 \%)$ & $2(16,67 \%)$ & $1(14,29 \%)$ & $13(24,07 \%)$ \\
\hline Normal & $1(16,67 \%)$ & $5(35,71 \%)$ & $4(26,67 \%)$ & $3(25 \%)$ & $3(42,85 \%)$ & $16(29,63 \%)$ \\
\hline Maigre & $3(50 \%)$ & $2(14,29 \%)$ & $2(13,33 \%)$ & $2(16,67 \%)$ & $1(14,29 \%)$ & $10(18,52 \%)$ \\
\hline \multicolumn{7}{|c|}{ Par rapport à TT } \\
\hline Obèse & $2(33,33 \%)$ & $11(78,57 \%)$ & $12(80 \%)$ & $10(83,33 \%$ & $5(71,43 \%)$ & $40(74,07 \%)$ \\
\hline Non obèse & $4(66.67 \%)$ & $3(21,43 \%)$ & $3(20 \%)$ & $2(16,67 \%)$ & $2(28,57 \%)$ & $14(25,93 \%)$ \\
\hline
\end{tabular}

Les résultats obtenus chez les hommes indiquent que 47,69\% des diabétiques hospitalisés sont en surcharge pondérale, $35,88 \%$ ont un poids normal et $16,92 \%$ de maigres selon l'IMC; La prévalence du surpoids augmente avec l'âge, plus importante entre 40 et 59 ans $(52,94 \%$ et $55 \%)$ puis chute à partir de 60 ans. Et par rapport à TT, Selon les critères de la FID, 49,23\% des hommes diabétiques ont une obésité abdominale; Sa 
prévalence est aussi importante entre 40 et 59 ans $(64,70 \%$ et $60 \%)$ puis baisse à partir de 60 ans.

Les résultats obtenus chez les femmes indiquent que 51,85\% des femmes diabétiques sont en surcharge pondérale, $29,63 \%$ ont un poids normal, et 18,52\% de maigres selon 1'IMC; La prévalence du surpoids augmente selon l'âge, surtout à partir de 40 ans, et baisse à partir de 60 ans. Et par rapport au TT, selon les critères de la FID 74,07 \% des femmes diabétiques échantillonnées ont une obésité abdominale ; La fréquence est plus importante à partir de 40 ans à toutes les tranches d'âges.

Tableau 3 : Etude du statut pondéral des deux sexes confondus en fonction de la tranche

\begin{tabular}{|c|c|c|c|c|c|c|}
\hline $\begin{array}{l}\text { Age } \\
\text { (ans) }\end{array}$ & $\leq 39$ & $40-49$ & $\frac{\text { a age }}{50-59}$ & $60-69$ & $\geq 70$ & Total \\
\hline \multicolumn{7}{|c|}{$\mathrm{P} / \mathrm{r}$ à l'IMC } \\
\hline Obésité & 0 & $\begin{array}{c}4 \\
(12,90 \%)\end{array}$ & $\begin{array}{c}10 \\
(28,57 \%) \\
\end{array}$ & $\begin{array}{c}5 \\
(21,74 \%)\end{array}$ & $\begin{array}{c}2 \\
(11,11 \%)\end{array}$ & $\begin{array}{c}21 \\
(17,65 \%)\end{array}$ \\
\hline Surpoids & $\begin{array}{c}4 \\
(36,36 \%)\end{array}$ & $\begin{array}{c}12 \\
(38,71 \%)\end{array}$ & $\begin{array}{c}10 \\
(28,57 \% \\
\end{array}$ & $\begin{array}{c}6 \\
(26,09 \%) \\
\end{array}$ & $\begin{array}{c}6 \\
(33,33 \%) \\
\end{array}$ & $\begin{array}{c}38 \\
(31,93 \%)\end{array}$ \\
\hline Normal & $1(9,09 \%)$ & $\begin{array}{c}12 \\
(33,33 \%) \\
\end{array}$ & $\begin{array}{c}11 \\
(31,42 \%) \\
\end{array}$ & $\begin{array}{c}7 \\
(30,43 \%) \\
\end{array}$ & $\begin{array}{c}7 \\
(38,89 \%) \\
\end{array}$ & $\begin{array}{c}38 \\
(31,93 \%) \\
\end{array}$ \\
\hline Maigre & $\begin{array}{c}7 \\
(54,55 \%) \\
\end{array}$ & $3(8,33 \%)$ & $\begin{array}{c}4(11,43 \\
\%)\end{array}$ & $\begin{array}{c}5 \\
(21,74 \%) \\
\end{array}$ & $\begin{array}{c}3 \\
(16,67 \%) \\
\end{array}$ & $\begin{array}{c}22 \\
(18,49 \%) \\
\end{array}$ \\
\hline \multicolumn{7}{|c|}{$\mathrm{P} / \mathrm{r}$ à TT } \\
\hline Obèse & $\begin{array}{c}2 \\
(16,67 \%)\end{array}$ & $\begin{array}{c}22 \\
(70,97 \%)\end{array}$ & $\begin{array}{c}24 \\
(68,57 \%) \\
\end{array}$ & $\begin{array}{c}13 \\
(56,52 \%)\end{array}$ & $\begin{array}{c}11 \\
(61,11 \%)\end{array}$ & $\begin{array}{c}72 \\
(60,50 \%)\end{array}$ \\
\hline $\begin{array}{l}\text { Non } \\
\text { obèse }\end{array}$ & $\begin{array}{c}10 \\
(83,33 \%) \\
\end{array}$ & $\begin{array}{c}9 \\
(29,03 \%) \\
\end{array}$ & $\begin{array}{c}11 \\
(31,43 \%) \\
\end{array}$ & $\begin{array}{c}10 \\
(43,48 \%)\end{array}$ & $\begin{array}{c}7 \\
(38,89 \%) \\
\end{array}$ & $\begin{array}{c}47 \\
(39,50 \%) \\
\end{array}$ \\
\hline
\end{tabular}

De manière globale, selon l'IMC 49,60 \% des 119 diabétiques pris au hasard sont en surcharge pondérale (Surpoids et obésité). La prévalence augmente avec l'âge, mais plus importante à partir de 40 ans. Et par rapport au TT, selon les critères de la FID, 60,50 \% des diabétiques échantillonnés présentent une obésité abdominale. La prévalence augmente aussi avec l'âge, mais elle est importante à partir de 40 ans à toutes les tranches d'âge, surtout entre 40 et 59 ans.

Tableau 4 : Etude des complications du diabète entraînant une hospitalisation

\begin{tabular}{|c|c|c|c|c|c|}
\hline \multirow{2}{*}{ Complications } & \multicolumn{3}{|c|}{ Complications spécifiques } & \multirow{2}{*}{\multicolumn{2}{|c|}{ Total }} \\
\hline & \multicolumn{2}{|r|}{ Hommes } & \multirow{2}{*}{$\begin{array}{c}\text { Femmes } \\
\text { Amputé : } 4(9,52 \%)\end{array}$} & & \\
\hline \multirow{7}{*}{$\begin{array}{c}\text { Macroangipathi } \\
\text { es }\end{array}$} & \multirow{3}{*}{$\begin{array}{l}\text { Avec } \\
\text { HTA }\end{array}$} & PD : $6(11,54 \%)$ & & \multirow{3}{*}{$\begin{array}{c}15 \\
(15,96 \%)\end{array}$} & \multirow{6}{*}{$\begin{array}{c}57 \\
(60,63 \\
\%)\end{array}$} \\
\hline & & Coma : $1 \quad(1,92 \%)$ & Coma: $2(4,76 \%)$ & & \\
\hline & & & Cœur : $2(4,76 \%)$ & & \\
\hline & \multirow{4}{*}{$\begin{array}{l}\text { Sans } \\
\text { HTA }\end{array}$} & PD : $12(23,08 \%)$ & PD : $5(11,90 \%)$ & \multirow{4}{*}{$\begin{array}{c}42 \\
(44,68 \%)\end{array}$} & \\
\hline & & $\begin{array}{c}\text { Amputé : } 9(17,31 \\
\%)\end{array}$ & $\begin{array}{c}\text { Pneumopathie : } 1 \\
(2,38 \%)\end{array}$ & & \\
\hline & & Coma : $6(11,54 \%)$ & Coma: $6(9,68 \%)$ & & \\
\hline & & AVC : $2(3,86 \%)$ & AVC : $1(2,38 \%)$ & & \\
\hline
\end{tabular}




\begin{tabular}{|c|c|c|c|c|c|}
\hline & & Cœur : $5(9,62 \%)$ & Couur : 5 (11,90\%) & & \\
\hline \multirow{8}{*}{$\begin{array}{c}\text { Microangiopathi } \\
\text { es }\end{array}$} & \multirow{3}{*}{$\begin{array}{l}\text { Avec } \\
\text { HTA }\end{array}$} & DE : $1(1,92 \%)$ & $\begin{array}{c}\text { Infection cutané : } 1(2,38 \\
\%)\end{array}$ & \multirow{3}{*}{$\begin{array}{c}9 \\
(9,57 \%)\end{array}$} & \multirow{8}{*}{$\begin{array}{c}38 \\
(39,27 \\
\%)\end{array}$} \\
\hline & & Coma: $3(7,14 \%)$ & Coma : $3(7,14 \%)$ & & \\
\hline & & PN : $1 \quad(1,92 \%)$ & & & \\
\hline & \multirow{5}{*}{$\begin{array}{l}\text { Sans } \\
\text { HTA }\end{array}$} & DE : $4(7,69 \%)$ & Coma : $5(11,90 \%)$ & \multirow{5}{*}{$\begin{array}{c}29 \\
(30,85 \%)\end{array}$} & \\
\hline & & Coma : $9(17,31 \%)$ & Cécité : $2(4,76 \%)$ & & \\
\hline & & IRC : $1(1,92 \%)$ & PN : $5(11,90 \%)$ & & \\
\hline & & PN : $2(3,86 \%)$ & & & \\
\hline & & $\begin{array}{c}\text { Pulmonaire : } 1(1,92 \\
\%)\end{array}$ & & & \\
\hline
\end{tabular}

94 diabétiques soumis à notre étude présentent des complications dégénératives soit $81,51 \%$ des diabétiques. C'est sur ces 94 diabétiques que s'est portée cette analyse.

Les résultats montrent que $60,63 \%$ présentent des macroangiopathies (complications dues à l'altération des artères de gros calibres) contre $39,17 \%$ qui présentent des microangiopathies (complications dues à l'altération des artères de petits calibres).

La prévalence des complications chez les malades sans HTA est plus élevée aussi bien dans les macroangiopathies que dans les microangiopathies.

\section{Discussion}

Les résultats recueillis chez les diabétiques hospitalisés du CHU de Yopougon, présentent trois types de diabètes qui sont le diabète type 1, le diabète type 2 et le diabète pancréatique, avec une forte prévalence de $\mathrm{D} 2$ $(95,80 \%)$. Comme partout dans le monde, le diabète de type 2 est la forme prédominante en Afrique subsaharienne avec plus de $90 \%$ des cas de diabète (Lokrou, Kouame, Cuisinier-Raynal, Papoz, and Darracq, 1994 ; Lokrou and Alléchi, 1995). Le D1 et le diabète pancréatique sont faiblement présents. Ce qui est en accord avec des travaux antérieurs de l'ADA qui parle de formes plus rares (ADA 2011). L'âge des diabétiques de type 2 étudiés varie de 29 à 81 ans avec une moyenne de 55,58 ans. Le D2 est alors présent dans la population de moins de 40 ans. Incontestablement, en Afrique, bien que le diabète de type 2 soit plus fréquent dans les populations âgées, il affecte de plus en plus de jeunes et d'adultes actifs de 20 à 40 ans (FID, 2009; Duboz, Chapuis-Lucciani, Boetsch, and Gueye, 2012). En plus, cette maladie est présente au niveau de toutes les catégories socio-économiques, que ce soit les classes aisées ou pauvres. En effet 42,02 \% des diabétiques soumis à notre étude sont à revenu faible et $57,98 \%$ à revenu élevé. Chez les femmes, la majorité sont à revenus faibles $(72,22 \%$ des femmes) et ont un niveau d'instruction bas (62,18\% des femmes) alors que chez les hommes la plupart ont des revenus élevés et sont instruits. Ce qui pourrait être expliquée 
par le fait que dans nos sociétés traditionnelles, la prise de poids est un facteur de bien-être et de beauté (Nono, Fokumlah, Sab Fru, Sabngwi, and Kengne, 2010). Signalons aussi que $32 \%$ des femmes D2 ont des antécédents de macrosomie. La macrosomie est en effet un facteur de risque du diabète chez les femmes. Les résultats montrent qu'il y a plus d'hommes diabétiques que de femmes $(56,64 \%$ vs $43,86 \%)$. Ces résultats sont en accord avec des travaux antérieurs de Oga et al. (2006). Cependant, les travaux de Duboz montrent une prévalence plus élevée chez les femmes que chez les hommes (Duboz et al., 2012). L'étude de l'obésité des diabétiques nous indique une forte prévalence de l'obésité centrale dans cette population $(60,50 \%)$. Tel que mentionné dans l'introduction, il existe donc un lien étroit entre diabète de type 2 et la surcharge pondérale (Mokdad et al., 2001). La prévalence de l'obésité est plus accentuée chez les femmes que chez les hommes $(74,07 \%$ vs 49,23\%). Ce qui souligne donc que beaucoup de femmes sont encore ignorantes des facteurs de risques et des conséquences du surpoids et de l'obésité sur la santé. De plus, la prise de poids semble être un indicateur de bien être en particulier chez les femmes mariées selon les croyances (Nono et al., 2010). En effet, plusieurs études démontrent que l'obésité est non seulement un critère de beauté mais aussi souvent perçue comme un signe de richesse et même de bonne santé. L'obésité en particulier abdominale ou androïde est reconnu comme un important facteur de risque des maladies métaboliques notamment du diabète de type 2 . Selon des études, la prévalence de l'obésité chez les personnes diabétiques de type 2 varierait de 14 à $35 \%$ (Lokrou and Gnangbomou-Boye, 1991 ; Gning et al., 2007). Cela se confirme dans cette étude où nous avons 17,65 \% d'obèses.

L'étude des complications montre un taux élevé des complications macrovasculaires $(60,63 \%)$ par rapport aux complications microvasculaires $(39,17 \%)$. Selon certaines études, les atteintes macrovasculaires seraient moins fréquentes en Afrique qu'en Occident, toutefois leur incidence croît actuellement en raison du changement dans le mode de vie des populations (Mbanya and Sobngwi, 2003 ; Kengne, Amoah, and Mbanya, 2005 ; Steyn et al., 2005; Ntyintyane, Panz, Raal et Gill, 2006). Selon la littérature, Il y a un lien entre l'hypertension artérielle et le diabète de type 2. En effet, les hypertendus développent plus souvent le diabète de type 2 que les normohypertendus, et réciproquement les patients atteints de D2 sont plus souvent hypertendus. En plus, en Afrique subsaharienne, l'HTA est très commune chez les patients diabétiques (N'Gouin-Claih et al. 2003). De nombreuses études ont montré que l'hypertension artérielle est non seulement plus fréquente, mais aussi plus précoce et plus sévère chez les personnes de race noire (Cooper and Rotimi, 1997). Pourtant, dans notre étude, la prévalence des patients sans HTA est beaucoup plus élevée 75,53\%. Aussi, 
les complications sont plus accentuées chez les patients sans HTA que chez les patients avec HTA. Cela peut s'expliquer par le fait que les données dans cette étude ont été recueillies chez les malades arrivés en urgence à 1'hôpital donc en général pas suivis. En effet, la majorité (78\%) des personnes atteintes de diabète n'ont pas encore été diagnostiquées (FID, 2011). Dans la plupart des cas, les patients se présentent avec l'une des complications du diabète et ou une comorbidité (FID, 2006). La plupart des HTA ont un suivi médical, sont donc soumis à un régime diététique (premier médicament dans le traitement du diabète). Car le régime alimentaire reste le moyen thérapeutique le moins onéreux et le plus facile à mettre en place pour l'ensemble des patients diabétiques.

Dans tous les cas, nous avons observé dans cette étude une forte fréquence de coma diabétique. Du fait qu'en Afrique, tous les accidents métaboliques du diabète, notamment les acidocétoses et les comas hyperosmolaires, sont souvent observés et très souvent irrécupérables en raison d'une prise en charge trop tardive, de la fréquence des infections, de l'arrêt du traitement, de la méconnaissance du diabète et du manque d'infrastructures pour une prise en charge adéquate (Pichard, Berthé, Traoré and Dembélé, 1988 ; Rolfe, Ephraim, Lincoln and Huddle, 1995). Dans les complications macrovasculaires, nous avons observé en plus des comas diabétiques, une forte fréquence de pied diabétique $(23,4 \%)$ et d'amputés $(13,82 \%)$. Des coronopathies, des AVC, des infections pulmonaires telles les pneumopathies sont aussi observées. Dans les complications microvasculaires nous observons des PN tels les DE, également des infections cutanées et pulmonaires, des insuffisances rénales chroniques, des rétinopathies (cécité). Le diabète pose donc un vrai problème de santé publique par le biais des complications chroniques dominées par les complications cardiovasculaires, le pied diabétique, l'insuffisance rénale chronique et la rétinopathie (Sidibé, Besançon and Beran, 2007).

\section{Conclusion}

Cette étude sur les complications du diabète chez les patients hospitalisés en urgence médicale nous a montré que 95,80\% des diabétiques sont des diabétiques du type 2. Dans cette étude, la grande majorité des malades ont une obésité abdominale. Cependant elle est beaucoup plus importante chez les femmes. Cette étude nous a également montré un fort taux de complications macrovasculaires, et une HTA non prédominante chez les diabétiques ayant des complications macrovasculaires que chez ceux ayant des complications microvasculaires. Il a été noté de façon spécifique que les patients avec complications macrovasculaires souffrent généralement de comas, de pied diabétiques, des amputations, des accidents vasculaires cérébraux (AVC), et que ceux avec les complications microvasculaires 
souffrent de disfonctionnements érectiles, d'insuffisances rénales chroniques, de polyneuropathies, d'infections pulmonaires et également de comas.

\section{References :}

American Diabetes Association (ADA) (2011). Standards of medical care in diabetes.

Diabetes Care, 34 (1), S11-S61.

ADA (2012). Diagnosis and Classification of Diabetes Mellitus. Diabetes care, 35(1),

S64-S71.

Cooper R. et Rotimi C. (1997). Hypertension in blacks, Am J Hypertens., 10 (7 Pt 1), 804- 812.

Duboz P., Chapuis-Lucciani N., Boetsch G. et Gueye L. (2012). Prevalence of diabetes and associated risk factors in a Senegalese urban (Dakar) population. Diabetes Metab.

38 (4) , 332-336.

Drouin, P., Blickle, J. F., Charbonnel, B., Eschwege, E., Guillausseau, P. J., Plouin, P. F., Daninos, J .M., Balarac, N., Sauvanet, J. P. (1999). Diagnostic et Classification du Diabète sucré : Les nouveaux critères. Diabetes and Metabolism, 25 , 72-83.

Fédération Internationale du Diabète (FID) (2009). Atlas du Diabète 4ed. Brussels. Fédération

Internationale du Diabète : Belgium.

FID (2011). Atlas du Diabète 5ed. Brussels. Fédération Internationale du Diabète : Belgium.

FID (2013). Atlas du Diabète 6ed. Brussels. Fédération Internationale du Diabète : Belgium.

FID (2006). Guide de prise en charge du diabète de type 2 pour l'Afrique subsaharienne,

Zanzibar-Tanzanie. Fédération Internationale du Diabète : Région Afrique, 47.

Gning, S. B., Thiam, M., Fall, F., Ba-Fall, K., Mbaye, P. S. et Fourcade, L. (2007). Le diabète sucré en Afrique subsaharienne: Aspects épidémiologiques, difficultés de prise en charge. Med. Trop., 67 (6) , 607611.

Guillausseau, P. J. (1997). Classification et critères diagnostiqués du diabète : propositions de

1'ADA et de l'OMS. Diabetes and Metabolism, 23 , 454-455.

Halimi S. (2003). Le diabète de type 2 ou diabète non insulino- dépendant (DNID) (223b).

Retrieved from Faculté de Médecine de Grenoble, Corpus Médical web site : 
http://www-sante.ujf-

grenoble.fr/SANTE/corpus/disciplines/endoc/diabeto/233b/leconimprim.pdf. Harris M, and Zimmet P. (1997). Classification of diabetes mellitus and other categories of glucose intolerance, in K. Alberti, P. Zimmet, and R. Defronzo (eds). International Textbook of Diabetes Mellitus, Second Edition (pp. 9-23), Chichester: John Wiley and Sons Ltd,.

Item 233C (2011). Complications dégénératives et métaboliques du diabète. Retrieved from Université Médicale Virtuelle Francophone, Support de Cours web site :

http://campus.cerimes.fr/endocrinologie/enseignement/item233c/site/html/1. html

Kengne, A. P., Amoah, A. G. B. et Mbanya, J. C. (2005). Cardiovascular complications of diabetes mellitus in sub-Saharan Africa. Circulation, 112 (23) , 3592-3601.

Lokrou, A. et Alléchi, C. (1995). Formes cliniques du diabète sucré en Côte d'Ivoire : Étude typologique de 1000 cas. Revue française d'endocrinologie clinique, nutrition et métabolisme, 36 (6) , 557-562.

Lokrou A. et Gnangbomou-Boye J. (1991). Le diabète non insulinodépendant en Côte d'Ivoire: Etude de 3950 cas. Revue française d'endocrinologie clinique, nutrition et métabolisme, 32 (6) , 581-587.

Lokrou, A., Kouame, P., Cuisinier-Raynal, J. C., Papoz, L. et Darracq, R. (1994). Typologie du diabète sucré en Côte-d'Ivoire: place du diabète tropical. Revue française d'endocrinologie clinique, nutrition et métabolisme, 35 (3) , 219-225.

Lokrou, A., Toutou, T., Ouedrago, Y., Groga-Bada, N., Koutouan, A., Diallo A., Niamkey, E., Soubeyrand, J., Beda, B. Y., (1987). Complication du diabète sucré en milieu hospitalier en Côte d'Ivoire. Médecine d'Afrique noire, 34 (7), 593-602.

Mbanya, J. C. et Sobngwi, E. (2003). Diabetes in Africa : Diabetes microvascular and macrovascular disease in Africa. J Cardiovasc Risk, 10 (2) , 97-102.

Mokdad, A. H., Bowman, B. A., Ford, E. S., Vinicor, F., Marks, J. S. et Koplan, J. P. (2001).

The continuing epidemics of obesity and diabetes in the United States. JAMA,

286 (10) , 1195-1200.

N'Gouin-Claih, A., Donzo, M., Barry, A., Diallo, A., Kabiné, O., Barry, R., Abdoulaye, K., Sylla, C., Magassouba, F. et Baldé, A. (2003). Prevalence of hypertension in Guinean rural areas, Arch. Mal. Coeur Vaiss., 96 (7-8) , 763 767.

Nono, C., Fokumlah, P., Sab Fru, C., Sabngwi, E. et Kengne, P. (2010). Prévalence de l'obèsité chez les femmes adultes en milieu urbain en Afrique 
sub-saharienne : le cas de limbé au cameroun. Elsevier Masson, 30 (1), A114

Ntyintyane, L. M., Panz, V. R., Raal, F. J. et Gill G. V. (2006). Metabolic syndrome, undiagnosed diabetes mellitus and insulin resistance are highly prevalent in urbanised South African blacks with coronary artery disease. Cardiovasc. J. S. Afr., 17 (2) , 50- 55.

Oga, A. S. S., Tebi, A., Aka, J., Adouéni, K. V., Malan, K. A., Kouadio, L. P. et Lokrou, A. (2006). Le diabète sucré diagnostiqué en Côte d'Ivoire : des particularités épidémiologiques. Med. Trop., 66 (3), 241-246.

Pichard, E., Berthé, G., Traoré, H. A. et Dembélé, M. (1988). Les acidocétoses diabétiques au Mali : A propos de vingt cas. Ann. Soc. bel. Méd. trop., $68,68-72$.

Rolfe, M., Ephraim, G. G., Lincoln, D. C. et Huddle K. R. (1995). Hyperosmolar non-ketotic diabetic coma as a cause of emergency hyperglycemic admission to Baragwanath hospital. S Aîr Usé , 85 (3) , 173176.

Sidibé, A., Besançon, S. et Beran, D. (2007). Le diabète : un nouvel enjeu de santé publique pour les pays en voie de développement : l'exemple du Mali. Médecine des maladies

Métaboliques, 1 (1) , 93-98. Steyn, K., Sliwa, K., Hawken, S., Commerford, P., Onen, C, Damasceno, A., Ounpuu, S. et Yusuf, S. (2005). Risk factors associated with myocardial infarction in Africa: the INTERHEART Africa study. Circulation, 112 (23), 3554-3561. 